\title{
Brain Activation Changes While Walking in Adults with and without Neurological Disease: Systematic Review and Meta-Analysis of Functional Near-Infrared Spectroscopy Studies
}

\author{
Alka Bishnoi ${ }^{1}$, Roee Holtzer ${ }^{2,3}$ and Manuel E. Hernandez ${ }^{1, *(D)}$ \\ 1 Department of Kinesiology \& Community Health, University of Illinois at Urbana-Champaign, \\ Urbana, IL 61801, USA; abishn2@illinois.edu \\ 2 Ferkauf Graduate School of Psychology, Yeshiva University, Bronx, NY 10461, USA; roee.holtzer@yu.edu \\ 3 Department of Neurology, Albert Einstein College of Medicine, Bronx, NY 10461, USA \\ * Correspondence: mhernand@illinois.edu; Tel.: +1-217-244-8971
}

check for

updates

Citation: Bishnoi, A.; Holtzer, R.; Hernandez, M.E. Brain Activation Changes While Walking in Adults with and without Neurological Disease: Systematic Review and Meta-Analysis of Functional Near-Infrared Spectroscopy Studies. Brain Sci. 2021, 11, 291. https:// doi.org/10.3390/brainsci11030291

Academic Editor: Hasan Ayaz

Received: 24 December 2020

Accepted: 23 February 2021

Published: 26 February 2021

Publisher's Note: MDPI stays neutral with regard to jurisdictional claims in published maps and institutional affiliations.

Copyright: (c) 2021 by the authors. Licensee MDPI, Basel, Switzerland. This article is an open access article distributed under the terms and conditions of the Creative Commons Attribution (CC BY) license (https:/ / creativecommons.org/licenses/by/ $4.0 /)$.

\begin{abstract}
Functional near-infrared spectroscopy (fNIRS) provides a useful tool for monitoring brain activation changes while walking in adults with neurological disorders. When combined with dual task walking paradigms, fNIRS allows for changes in brain activation to be monitored when individuals concurrently attend to multiple tasks. However, differences in dual task paradigms, baseline, and coverage of cortical areas, presents uncertainty in the interpretation of the overarching findings. (2) Methods: By conducting a systematic review of 35 studies and meta-analysis of 75 effect sizes from 17 studies on adults with or without neurological disorders, we show that the performance of obstacle walking, serial subtraction and letter generation tasks while walking result in significant increases in brain activation in the prefrontal cortex relative to standing or walking baselines. (3) Results: Overall, we find that letter generation tasks have the largest brain activation effect sizes relative to walking, and that significant differences between dual task and single task gait are seen in persons with multiple sclerosis and stroke. (4) Conclusions: Older adults with neurological disease generally showed increased brain activation suggesting use of more attentional resources during dual task walking, which could lead to increased fall risk and mobility impairments. PROSPERO ID: 235228.
\end{abstract}

Keywords: neuroimaging; dual-task walking; neurological disorders

\section{Introduction}

Daily living activities such as walking rely on multiple neuronal structures [1]. Walking involves complex interactions between different cortical areas of the brain, which regulate attention and executive function, to avoid obstacles and safely navigate complex environments [2,3]. This ability to process and navigate properly can become compromised with age or in adults with neurological or musculoskeletal disorders [4].

Brain activity or cortical activity can be measured by various neuroimaging methods such as functional magnetic resonance imaging (fMRI), magnetoencephalography (MEG), positron-emission tomography (PET), electroencephalography (EEG), and functional near-infrared spectroscopy (fNIRS). Although fMRI is considered gold standard for the assessment of activity in cortical areas, it suffers from the susceptibility of motion artifacts and restrictions in doing walking activities [5-7]. Similarly, MEG exhibits high vulnerability towards motion artifacts [6], while PET doesn't allow repeated measurement due to injection of radioactive tracers [8]. On the other hand, EEG has weak spatial resolution, takes time to prepare, is vulnerable to artifacts and signal processing is difficult for nonexperts in the field [9-11]. Due to these restrictions among neuroimaging techniques, Functional Near Infrared Spectroscopy (fNIRS) has been used to record the cortical activity 
while walking. fNIRS is an optical neuroimaging technique for assessing cortical activity through the hemodynamic response of the brain while the participant walks freely [12], especially while dual task walking.

A dual task (DT) paradigm is a behavioral procedure in which individuals are required to perform two tasks simultaneously. Dual tasks can involve a wide range of concurrent sensory, motor, or cognitive tasks, and may be analogous to activities in daily life such as cooking, shopping, or walking while talking. Despite such variety, dual tasking often results in decline in performance in one component or both components of the dual task as compared to the single task, which is called dual task interference or dual task cost. The concept of capacity limitation in cognitive resource and performance limitation have been used to explain this dual task interference [13]. DT paradigms have received significant interest among researchers as it provides a gold standard for evaluation of the "central executive system" [14,15]. Moreover, in the context of gait, the use of DT paradigms allows researchers to assess the causal effect attentional resources have on walking performance under circumstances that better approximate real life conditions [16,17]. In comparison with a single task condition, DT conditions have shown activation in the dorsolateral prefrontal cortex of the brain [14,18]. This pattern of increased cortical activation in the prefrontal cortex has also been demonstrated through fNIRS studies of dual task walking.

There seems to be considerable amount of literature on fNIRS measuring cortical activity in areas such as the PFC, Pre-Motor Cortex (PMC), Supplementary Motor Area (SMA), and Sensory Motor Cortex (SMC) while performing dual tasks. However, previous fNIRS reviews have described the history of fNIRS [19], modelling and analysis of fNIRS [20,21], comparison of patterns of cortical activity using a variety of imaging techniques in walking studies [1,4], methodological approaches in postural and walking studies [22], data processing techniques [23], and PFC activations measured during walking [24] or during cognitive and motor tasks [25]. However, no review has undertaken a quantitative synthesis of brain activation differences in adults while dual task walking and how these effects differ among adults with and without neurological diseases.

Given the increased usage of fNIRS in recent years to study cortical control of locomotion, this study was designed to address an important gap in the literature: What are the brain activation differences while dual task walking in adults with neurological diseases? Specifically, we systematically reviewed and quantitatively synthesize brain activation differences, assessed using fNIRS, in adults with and without neurological disease while dual-task walking. The aims of this systematic review were to: (1) Quantify the changes in cortical activation patterns between different dual tasks; (2) quantify activation differences between different populations; (3) evaluate each study based on the quality assessment criteria.

\section{Materials and Methods}

\subsection{Study Selection Criteria}

Studies that met all of the following criteria were included in the review: (1) Study design: Cross-sectional, randomized controlled trial (RCT), cohort study, pre-post study; (2) population: Adults 18 years and older with or without neurological disease; (3) measured walking in the dual task; (4) used fNIRS to quantify oxygenated hemoglobin $(\mathrm{HbO} 2)$ as the outcome measure; (5) was a peer-reviewed article; (6) was published in English; (7) Subject: Humans; and (8) Timespan: All years. Studies were excluded if: (1) fNIRS was not used; (2) dual task didn't involve walking; (3) conference proceeding or review article; or (4) was a non-English publication.

\subsection{Search Strategy}

The systematic review and meta-analysis described in the Preferred Reporting Items for Systematic Reviews and Meta-analysis process [26] were adopted to guide the review process. A keyword search was performed in PubMEd, Cumulative Index of Nursing and Allied Health, PsycINFO, Scopus and Web of Science from August 2019-June 2020. The 
search algorithm included all possible combinations of keywords from the five groups: (1) "Dual"; (2) "task" or "motor skill"; (3) "gait", "locomotion", "walking", "ambulation"; (4) "adults"; (5) "neuroimaging" or "fNIRS" or "functional near infra*". The specific search algorithm for each database is provided in Appendix A.

Titles and abstracts of the articles identified through the keyword search were screened for the study selection criteria. Two reviewers (A.B. and M.E.H.) independently conducted title and abstract screening to determine their eligibility. Interrater agreement was determined by Intraclass correlation coefficient value and authors showed excellent correlation $(I C C=0.84)$. Any disagreements were resolved by discussion. A cited reference search (i.e., forward reference search) and reference list search (i.e., backward reference search) were conducted on the full text articles that met the study eligibility criteria from the keyword search. Articles identified through forward and backward search were further screened and evaluated by using the same study selection criteria. Reference searches were repeated on all newly identified articles until no additional relevant articles were found.

\subsection{Data Extraction}

A standardized data extraction form was used to collect methodological and outcome variables from each selected study including author(s), year of publication, study design, sample size, participant characteristics (i.e., gender, age, pathology), single-task type, dualtask type, outcome measures $(\mathrm{HbO} 2, \mathrm{Hb}$ mean and $\mathrm{SD})$ and key findings in terms of effect of dual-task on walking in adults assessed by fNIRS was extracted.

\subsection{Quantitative Data Synthesis}

Meta-analysis was performed to estimate the pooled effect size for PFC activation, measured by the oxygenated hemoglobin $(\mathrm{HbO} 2)$, furthermore, we performed subgroup analysis on fNIRS-derived $\mathrm{HbO} 2$ assessed during active walking under single and dualtask conditions. Secondary meta-analysis was done to find activation differences between single task and dual task in adults with and without neurological diseases among various studies $($ DT difference $=$ DT mean-Single task mean). Several studies were excluded from meta-analysis because they didn't have control group in the study or dual task wasn't implemented in control group. Study heterogeneity was assessed using the $\mathrm{I}^{2}$ index. The level of heterogeneity represented by $\mathrm{I}^{2}$ was interpreted as modest $\left(\mathrm{I}^{2} \leq 25 \%\right)$, moderate $\left(25 \%<\mathrm{I}^{2} \leq 50 \%\right)$, substantial $\left(50 \%<\mathrm{I}^{2} \leq 75 \%\right)$ or considerable $\left(\mathrm{I}^{2}>75 \%\right)$ [27]. A fixed-model was estimated when modest to moderate heterogeneity was present, and a random-effect model was estimated when substantial to considerable heterogeneity was present [27]. Publication bias was assessed by a visual inspection of funnel plots and tested by Egger's tests. All statistical analyses were conducted using the Stata 14.2 SE version (StataCorp, College Station, TX, USA). All analysis used two-sided $t$-tests and $p$ values equal or less than 0.05 were considered statistically significant.

\subsection{Study Quality Assessment}

We used the National Institutes of Health's (NIH) Quality Assessment tool for Observational cohort and Cross-sectional studies to assess the quality of each included study [28] (https:/ / www.nhlbi.nih.gov/health-topics/study-quality-assessment-tools, accessed on 21 October 2019). The following questions were used for the criteria: (1) Was the research question or objective of the study clearly stated? (2) Was the study population clearly specified and defined? (3) Were the inclusion and exclusion criteria for being the study prespecified and uniformly applied to all the participants? (4) Was sample size justification, power description or variance and effect estimated provided? (5) For analysis, were the exposure of interest measured prior to the outcomes being measured? (6) Were the exposure measures clearly defined and valid? (7) Were the outcome measures clearly defined and valid? (8) Were potential confounding variables measured and adjusted statistically? (9) Was dual task clearly defined and applied? (10) Was fNIRS applied to prefrontal cortex in the study? This assessment tool rates each study based on score range 0 (unmet), 
1 (partially met), 2 (completely met). A study-specific global score ranging from 0 to 18 was calculated by summing up scores across all criteria. The study quality assessment helped measure the strength of scientific evidence but was not used to determine the inclusion of studies.

\section{Results}

\subsection{Study Selection}

As Figure 1 shows, a total of 61 articles were identified through keyword and reference search (forward/backward search), 23 of them were excluded in title and abstract screening. The remaining 38 articles were reviewed in full texts, and 3 of them is excluded for not meeting the study selection criteria as listed in Figure 1. The remaining 35 [29-63] articles were included in the review.
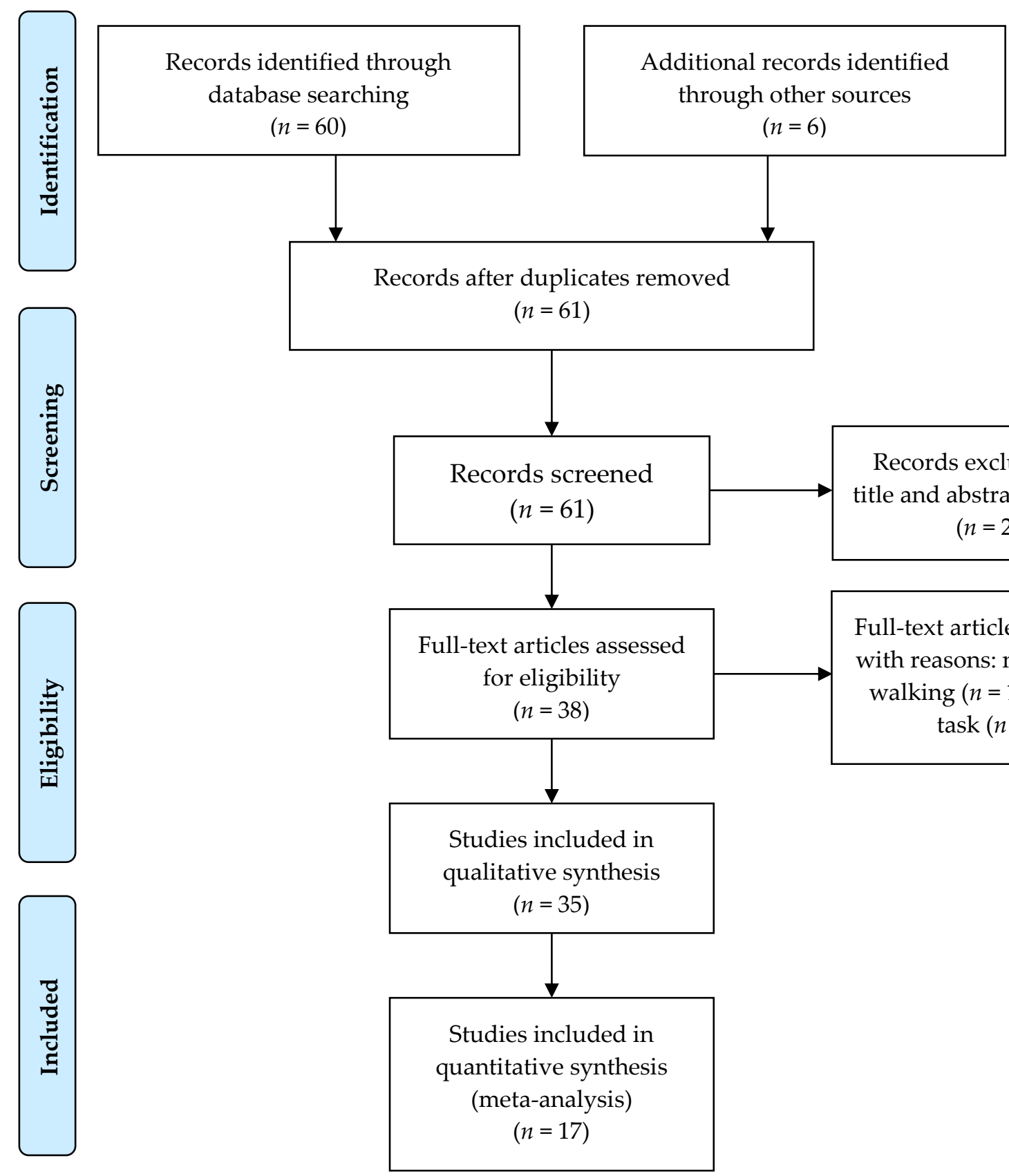

Records excluded after title and abstract screening

$$
(n=23)
$$

Full-text articles excluded, with reasons: no dual task walking $(n=1)$, no dual task $(n=2)$

Figure 1. Systematic review PRISMA flow diagram. 


\subsection{Basic Characteristics of Included Studies}

Tables 1 and 2 reports the data extraction of 35 articles included in the review. As per basic characteristics of the studies, there are 10 studies including 178 healthy young adults aged between 19-39 years old; and 24 studies, including 2184 participants aged $>55$ years old. In neurological population, there are 4 studies including 175 people with Parkinson's disease aged $>60$ years old; 5 studies including 100 people with Stroke aged $>52$ years old; and 3 studies, including 32 people with Multiple Sclerosis aged $>50$ years old. Lastly, there is only one study, including 16 people with Mild Cognitive Impairment aged $>70$ years old.

Table 1. Study and Participant characteristics.

\begin{tabular}{|c|c|c|c|c|c|c|c|}
\hline \multirow{2}{*}{ Study } & \multirow{2}{*}{ Study ID } & \multirow{2}{*}{ Year } & \multirow{2}{*}{ Type of Study } & \multicolumn{2}{|c|}{ Age (Mean \pm SD (years)) } & \multicolumn{2}{|c|}{ Population } \\
\hline & & & & HYA & HOA & HYA & HOA \\
\hline Beurskens et al. [41] & 1 & 2014 & Cross-sectional & $25 \pm 3$ & $71 \pm 4$ & 15 & 10 \\
\hline Chen et al. [57] & 2 & 2017 & Cross-sectional & NA & $78.1 \pm 6$ & NA & 90 \\
\hline Fraser et al. [59] & 3 & 2016 & Cross-sectional & $22 \pm 2$ & $67 \pm 5$ & 19 & 14 \\
\hline George et al. [60] & 4 & 2019 & Cross-sectional & NA & $76 \pm 7$ & NA & 325 \\
\hline Holtzer et al. ${ }^{\dagger}[35]$ & 5 & 2011 & Cross-sectional & $19-29$ & $69-88$ & 11 & 11 \\
\hline Holtzer et al. ${ }^{+}[36]$ & 6 & 2016 & Cross-sectional & NA & $74 \pm 6$ & NA & 167 \\
\hline Holtzer et al. [37] & 7 & 2016 & Cross-sectional & NA & $77 \pm 7$ & NA & 314 \\
\hline Holtzer et al. [34] & 8 & 2017 & Cross-sectional & NA & $77 \pm 7$ & NA & 318 \\
\hline Holtzer et al. [33] & 9 & 2018 & Cross-sectional & NA & $77 \pm 7$ & NA & 315 \\
\hline Holtzer et al. [31] & 10 & 2019 & Cross-sectional & NA & $78 \pm 6$ & NA & 75 \\
\hline Holtzer et al. ${ }^{\dagger}[32]$ & 11 & 2019 & Cross-sectional & NA & $78 \pm 6$ & NA & 83 \\
\hline Lin et al. [38] & 12 & 2016 & Cross-sectional & $20-27$ & NA & 24 & NA \\
\hline Lu et al. ${ }^{+}[40]$ & 13 & 2015 & Cross-sectional & $23 \pm 2$ & NA & 17 & NA \\
\hline Lucas et al. ${ }^{+}[42]$ & 14 & 2018 & Cross-sectional & NA & $75 \pm 5$ & NA & 55 \\
\hline Meester et al. [45] & 15 & 2014 & Cross-sectional & $28 \pm 6$ & NA & 17 & NA \\
\hline Metzger et al. [46] & 16 & 2017 & Cross-sectional & $28,19-39$ & NA & 12 & NA \\
\hline Mirelman et al. ${ }^{+}[48]$ & 17 & 2014 & Cross-sectional & $31 \pm 4$ & NA & 23 & NA \\
\hline Mirelman et al. ${ }^{+}[47]$ & 18 & 2017 & Cross-sectional & $31 \pm 4$ & $70 \pm 6$ & 23 & 20 \\
\hline Osofundiya et al. [51] & 19 & 2016 & Cross-sectional & NA & $81 \pm 7$ & NA & 20 \\
\hline Stuart et al. [54] & 20 & 2019 & Cross-sectional & $20 \pm 1$ & $73 \pm 8$ & 17 & 18 \\
\hline Verghese et al. [55] & 21 & 2017 & Cross-sectional & NA & $75 \pm 6$ & NA & 166 \\
\hline \multirow[t]{2}{*}{ Wagshul et al. [56] } & 22 & 2019 & Cross-sectional & NA & $>65$ & NA & 55 \\
\hline & & & & PD & HOA & PD & HOA \\
\hline Al-yahya et al. ${ }^{+}[29]$ & 23 & 2019 & Cross-sectional & $66 \pm 6$ & $60 \pm 7$ & 29 & 22 \\
\hline Maidan et al. ${ }^{+}[44]$ & 24 & 2016 & Cross-sectional & $72 \pm 1$ & $70 \pm 1$ & 68 & 38 \\
\hline Maidan et al. ${ }^{+}[43]$ & 25 & 2018 & RCT & $72 \pm 1$ & NA & 64 & NA \\
\hline \multirow[t]{2}{*}{ Nieuwhof et al. [50] } & 26 & 2016 & Cross-sectional & $71 \pm 5$ & NA & 14 & NA \\
\hline & & & & Stroke & HOA & Stroke & HOA \\
\hline Al-yahya et al. ${ }^{\dagger}[30]$ & 27 & 2016 & Cross-sectional & $60 \pm 15$ & $54 \pm 9$ & 19 & 20 \\
\hline Chatterjee et al. ${ }^{+}[63]$ & 28 & 2019 & Cross-sectional & $60 \pm 10$ & NA & 33 & NA \\
\hline Hermand et al. [61] & 29 & 2019 & Cross-sectional & $71 \pm 10$ & NA & 11 & NA \\
\hline Liu et al. [39] & 30 & 2018 & Cross-sectional & $52 \pm 11$ & NA & 23 & NA \\
\hline \multirow[t]{2}{*}{ Mori et al. [49] } & 31 & 2018 & Cross-sectional & $61 \pm 9$ & $66 \pm 1$ & 14 & 14 \\
\hline & & & & MS & HOA & MS & HOA \\
\hline Chaparro et al. ${ }^{\dagger}[52]$ & 32 & 2017 & Cross-sectional & $56 \pm 5$ & $63 \pm 4$ & 10 & 12 \\
\hline Hernandez et al. ${ }^{\dagger}[62]$ & 33 & 2016 & Cross-sectional & $57 \pm 5$ & $61 \pm 4$ & 8 & 8 \\
\hline \multirow[t]{2}{*}{ Saleh et al. [53] } & 34 & 2018 & Cross-sectional & $50 \pm 8$ & $50 \pm 9$ & 14 & 14 \\
\hline & & & & MCI & HOA & MCI & HOA \\
\hline Doi et al. ${ }^{+}[58]$ & 35 & 2013 & Cross-sectional & $75 \pm 7$ & NA & 16 & NA \\
\hline
\end{tabular}

Note: Values are in mean $\pm \mathrm{SD}$ or as otherwise indicated, ${ }^{+}$represents studies included in the meta-analysis. Abbreviations: $\mathrm{MCI}$, Mild cognitive impairment; MS, Multiple Sclerosis; PD, Parkinson's Disease; HOA, Healthy Older adults; HYA, Healthy Young Adults; RCT, Randomized Controlled Trial; NA, not available. 
Table 2. Oxyhemoglobin $\left(\mathrm{HbO}_{2}\right)$ \& Deoxyhemoglobin $(\mathrm{Hb})$ outcome measures for studies included in the review.

\begin{tabular}{|c|c|c|c|c|c|c|}
\hline \multirow[t]{2}{*}{ Study ID } & \multirow[t]{2}{*}{ Single Task } & \multicolumn{2}{|c|}{ Mean \pm SD } & \multirow[t]{2}{*}{ Dual Task } & \multicolumn{2}{|c|}{ Mean \pm SD } \\
\hline & & HYA & HOA & & HYA & HOA \\
\hline 01 & & $-0.13 \pm 0.02$ & $-0.09 \pm 0.04$ & $\begin{array}{c}\text { Walk \& visual check } \\
\text { WWT }\end{array}$ & $\begin{array}{l}-0.15 \pm 0.02 \\
-0.22 \pm 0.02\end{array}$ & $\begin{array}{l}-0.23 \pm 0.05 \\
-0.09 \pm 0.03\end{array}$ \\
\hline 02 & & NA & $0.30 \pm 1.21$ & $\begin{array}{l}\text { WWT } \\
\text { OW }\end{array}$ & NA & $1.08 \pm 1.51$ \\
\hline 03 & & NA & NA & n-back task & $\begin{array}{l}\text { 1-back: } 15.87 \pm 5.62 \\
\text { 2-back: } 13.67 \pm 9.39\end{array}$ & $\begin{array}{l}\text { 1-back: } 15.87 \pm 5.62 \\
\text { 2-back: } 13.67 \pm 9.39\end{array}$ \\
\hline 04 & & NA & $0.11 \pm 1.2 \diamond$ & RAL & NA & $0.705 \pm 1.28$ \\
\hline $05^{+}$ & & $0.43 \pm 0.83$ & $0.42 \pm 0.49$ & RAL & $1.96 \pm 1.27$ & $0.64 \pm 0.60$ \\
\hline $06^{+}$ & & NA & $0.22 \pm 2.02$ & RAL & NA & $0.94 \pm 2.28$ \\
\hline 07 & & NA & $0.11 \pm 1.25$ & RAL & NA & $0.73 \pm 1.41$ \\
\hline 08 & & NA & $0.11 \pm 0.65$ & RAL & NA & $0.66 \pm 0.86$ \\
\hline 09 & & NA & $0.11 \pm 0.64$ & RAL & NA & $0.7 \pm 0.88$ \\
\hline $11^{+}$ & & NA & $0.18 \pm 1.51$ & RAL & NA & $0.90 \pm 1.72$ \\
\hline 12 & & $\begin{array}{c}-0.06 \pm 0.26 \\
0.33 \pm 0.36 \\
-0.24 \pm 0.36\end{array}$ & NA & $\begin{array}{l}\text { n-back walking } \\
\text { Wide path } \\
\text { Narrow path } \\
\text { Obstacle path }\end{array}$ & $\begin{array}{c}\text { 1-back } \\
-0.92 \pm 0.33 \\
-0.47 \pm 0.35 \\
-1.05 \pm 0.39\end{array}$ & NA \\
\hline $13^{+}$ & & NR & NA & SS7s & NR & NA \\
\hline $14^{+}$ & & NA & $0.39 \pm 0.97$ & RAL & NA & $0.9 \pm 1.54$ \\
\hline 15 & & $\begin{array}{c}0.22 \pm 0.11 \\
-0.1 \pm 0.25^{\prime \prime} \\
\end{array}$ & NA & SS7s & $\begin{array}{c}0.36 \pm 0.1 \\
-0.15 \pm 0.30^{\prime \prime} \\
\end{array}$ & NA \\
\hline 16 & & NA & NA & Letter generation task & NA & NA \\
\hline $17^{\dagger}$ & & $0.02 \pm 0.03 \bullet$ & NA & $\begin{array}{c}\text { SS7s } \\
\text { Counting back }\end{array}$ & $\begin{array}{l}0.28 \pm 0.03 \\
0.18 \pm 0.03\end{array}$ & NA \\
\hline $18^{+}$ & & $-0.01 \pm 0.04 \diamond$ & $0.17 \pm 0.05 \bullet$ & $\begin{array}{l}\text { SS7s } \\
\text { OW }\end{array}$ & $\begin{array}{l}0.15 \pm 0.04 \bullet \\
0.11 \pm 0.04\end{array}$ & $\begin{array}{l}0.31 \pm 0.05 \bullet \\
0.28 \pm 0.07\end{array}$ \\
\hline
\end{tabular}


Table 2. Cont.

\begin{tabular}{|c|c|c|c|c|c|c|}
\hline \multirow[t]{2}{*}{ Study ID } & \multirow[t]{2}{*}{ Single Task } & \multicolumn{2}{|c|}{ Mean \pm SD } & \multirow[t]{2}{*}{ Dual Task } & \multicolumn{2}{|c|}{ Mean \pm SD } \\
\hline & & HYA & HOA & & HYA & HOA \\
\hline 19 & & NA & $0.36 \pm 0.40$ & $\begin{array}{l}\text { Recite alternate letters } \\
\text { Precision walking }\end{array}$ & NA & $\begin{array}{c}1.145 \pm 0.5 \\
1.595 \pm 0.445\end{array}$ \\
\hline 20 & & NA & NA & Digit vigilance task & $-0.001 \pm 0.07$ & $-0.011 \pm 0.07$ \\
\hline 21 & & NA & $0.08 \pm 0.62$ & RAL & NA & $0.74 \pm 0.85$ \\
\hline 22 & & NA & $0.4 \pm 1.04$ & RAL & NA & $1.03 \pm 1.58$ \\
\hline $23^{+}$ & & $\begin{array}{c}1.27 \pm 0.33 \\
-0.76 \pm 0.24\end{array}$ & $\begin{array}{c}1.10 \pm 0.49 \\
-0.82 \pm 0.36\end{array}$ & SS7s & $\begin{array}{c}1.87 \pm 0.46 \\
-0.98 \pm 0.34\end{array}$ & $\begin{array}{r}2.42 \pm 0.68 \\
-1.50 \pm 0.50 \bullet " \prime\end{array}$ \\
\hline $24^{+}$ & & $0.24 \pm 0.02 \diamond$ & $0.14 \pm 0.04 \bullet$ & $\begin{array}{l}\text { SS3s } \\
\text { OW }\end{array}$ & $0.33 \pm 0.03$ & $0.25 \pm 0.04 \bullet$ \\
\hline $25^{+}$ & & $\begin{array}{l}-0.04 \pm 0.035 \\
-0.04 \pm 0.035\end{array}$ & NA & $\begin{array}{l}\text { SS3s } \\
\text { OW }\end{array}$ & $\begin{array}{c}-0.015 \pm 0.035 \\
0.005 \pm 0.035\end{array}$ & NA \\
\hline \multirow[t]{2}{*}{26} & & NA & NA & $\begin{array}{l}\text { Counting forward } \\
\text { SS } \\
\text { Reciting digits }\end{array}$ & $\begin{array}{c}0.3 \pm 0.07 ; 0.00 \pm 0.05^{\prime \prime} \\
0.44 \pm 0.20 ;-0.02 \pm 0.04^{\prime \prime} \\
0.38 \pm 0.15 ;-0.1 \pm 0.19^{\prime \prime}\end{array}$ & NA \\
\hline & & Stroke & HOA & & Stroke & HOA \\
\hline $27^{\dagger}$ & & $\begin{array}{c}0.69 \pm 0.22 \\
-0.45 \pm 1.0\end{array}$ & $\begin{array}{l}0.49 \pm 0.13 \\
-0.4 \pm 1.0\end{array}$ & SS7s & $\begin{array}{c}1.02 \pm 0.28 \\
-0.65 \pm 1.0\end{array}$ & $\begin{array}{c}0.72 \pm 0.21 \\
-0.51 \pm 0.99\end{array}$ \\
\hline $28^{+}$ & & $\begin{array}{c}0.26 \pm 0.09 \\
-0.1 \pm 0.05 \text { औ" }\end{array}$ & NA & SS7s & $\begin{array}{l}0.92 \pm 0.17 \bullet \\
-0.2 \pm 0.1 \bullet " \prime\end{array}$ & NA \\
\hline 29 & & $\begin{array}{cc}\text { ST low } & 1.19 \pm 0.7 \\
\text { ST high } & 1.23 \pm 1.3 \\
\text { walk } & 2.42 \pm 1.93\end{array}$ & NA & N-back & $\begin{array}{cc}\text { DT low } & 2 \pm 2.24 \\
\text { DT high } & 2.69 \pm 2.22\end{array}$ & NA \\
\hline 30 & & $-5.3 \pm 1.7^{\bullet:}$ & NA & $\begin{array}{l}\text { SS3s } \\
\text { WMT }\end{array}$ & $18.67 \pm 2.1 \bullet:$ & NA \\
\hline $31^{\Delta}$ & & $-0.3 \pm 1.73$ & $0.69 \pm 2.11$ & SS3s & $-0.073 \pm 0.41$ & $2.08 \pm 1.87$ \\
\hline
\end{tabular}


Table 2. Cont

\begin{tabular}{|c|c|c|c|c|c|c|}
\hline \multirow[t]{2}{*}{ Study ID } & \multirow[t]{2}{*}{ Single Task } & \multicolumn{2}{|c|}{ Mean \pm SD } & \multirow[t]{2}{*}{ Dual Task } & \multicolumn{2}{|c|}{ Mean \pm SD } \\
\hline & & MS & HOA & & MS & HOA \\
\hline $32^{+}$ & & $0.39 \pm 0.1 \bullet$ & $0 \pm 0.06 \bullet$ & WWT & $0.92 \pm 0.1^{\bullet}$ & $0.13 \pm 0.06 \bullet$ \\
\hline \multirow[t]{2}{*}{34} & & $2.22 \pm 0.91 \bullet:$ & $0.16 \pm 0.95 \bullet:$ & SS7s & $1.64 \pm 0.95$ & $3.18 \pm 1.54$ \\
\hline & & MCI & HOA & & MCI & HOA \\
\hline $35^{+}$ & & $0.06 \pm 0.01 \bullet$ & NA & Letter fluency task & $0.16 \pm 0.02 \diamond$ & NA \\
\hline
\end{tabular}

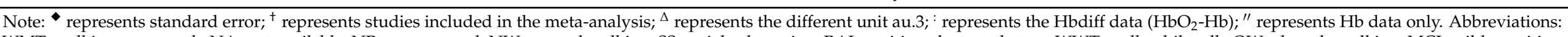

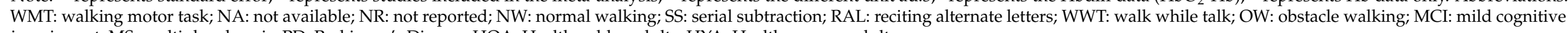
impairment; MS: multiple sclerosis; PD: Parkinson's Disease; HOA: Healthy older adults; HYA: Healthy young adults. 
Table 2 describes the oxyhemoglobin and deoxyhemoglobin outcomes of the studies while single task walking and dual task walking. There were 22 studies involving healthy young or older adults' data while doing dual task walking [31-38,40-42,45-48,51,54-57,59,60]; out of which 17 involve letter generation tasks as a cognitive task while walking [31-37,42,46, 51,55-57,60]; and 7 studies involve serial subtraction tasks while walking [38,39,45,47,48,54,59]; and 3 involved obstacle walking $[38,47,57]$. In addition, there were 4 studies showing PFC activation comparison between healthy older adults and people with Parkinson's disease while doing serial subtraction as a cognitive task while walking [29,43,44,50]; and 2 studies did obstacle walking $[43,44]$.

Appendix B provides further details on whether the study consisted of over-ground walking or treadmill walking, provided instruction for prioritization, or addressed systemic confounders.

\subsection{Meta-Analysis}

For overall meta-analysis, we did find increase in PFC activation among single task and dual task walking conditions, relative to standing baselines prior to the task. For normal walking, total number of studies included in the random effect model are 24; overall effect size was significant $(z=6.89 ; p<0.01)$, further, subgroup analysis showed that even normal walking can show significant increase in PFC activation among healthy older adults $(\mathrm{z}=4.51 ; p<0.01)$, people with stroke $(\mathrm{z}=2.06 ; p<0.05)$ and multiple sclerosis $(\mathrm{z}=2.64 ; p=0.008)$.

For dual task walking, we separated the meta-analysis for serial subtraction, obstacle walking and letter generation tasks. Overall, all tasks showed significant increase in PFC activation (Serial Subtraction, $\mathrm{z}=7.79 ; p<0.001$; Obstacle walking, $\mathrm{z}=4.52 ; p<0.001$; Letter generation, $\mathrm{z}=6.36 ; p<0.001)$. The effect of serial subtraction and letter generation task was highest among the three tasks (Figure 2).

For subgroup analysis in serial subtraction, a significant increased effect was found in all groups: healthy young adults $(z=3.89 ; p=0.001)$, healthy older adults $(z=4.24$; $p<0.001)$, people with Parkinson's disease $(z=3.79 ; p<0.01)$, people with stroke $(z=6.46$; $p<0.001)$. For subgroup analysis in obstacle walking, a significant increased effect was found in healthy older adults only $(\mathrm{z}=7.41 ; p<0.001)$. For subgroup analysis in letter generation task, a significant increased effect was found in healthy older adults $(\mathrm{z}=4.84$; $p<0.001)$ and people with multiple sclerosis $(z=3.16 ; p=0.002)$ (Figures 3 and 4$)$.

In addition, we performed a DT difference meta-analysis (DT diff = DT mean- ST mean) which showed that letter generation tasks $(\mathrm{z}=4.17, \mathrm{p}<0.01)$, serial subtraction $(\mathrm{z}=3.83$, $p<0.01)$, and obstacle walking $(\mathrm{z}=2.32, p=0.02)$ tasks demonstrated significantly increased PFC activation, in healthy older adults. In healthy young adults, serial subtraction tasks showed significant activation differences $(\mathrm{z}=4.28, p<0.01)$ and in adults with neurological diseases, letter generation tasks in persons with multiple sclerosis $(z=3.64, p<0.01)$ and serial subtraction in persons with stroke $(z=3.46, p<0.01)$ demonstrated significantly increased PFC activation, relative to single task walking (Figures 5 and 6).

Lastly, we did a publication bias analysis on the DT difference effect sizes. Egger's test indicated no presence of publication bias in healthy adults across all reported dual tasks $(n=15, p=0.095$, Figure 7$)$, nor in adults with neurological conditions across all tasks $(n=7, p=0.233)$. 


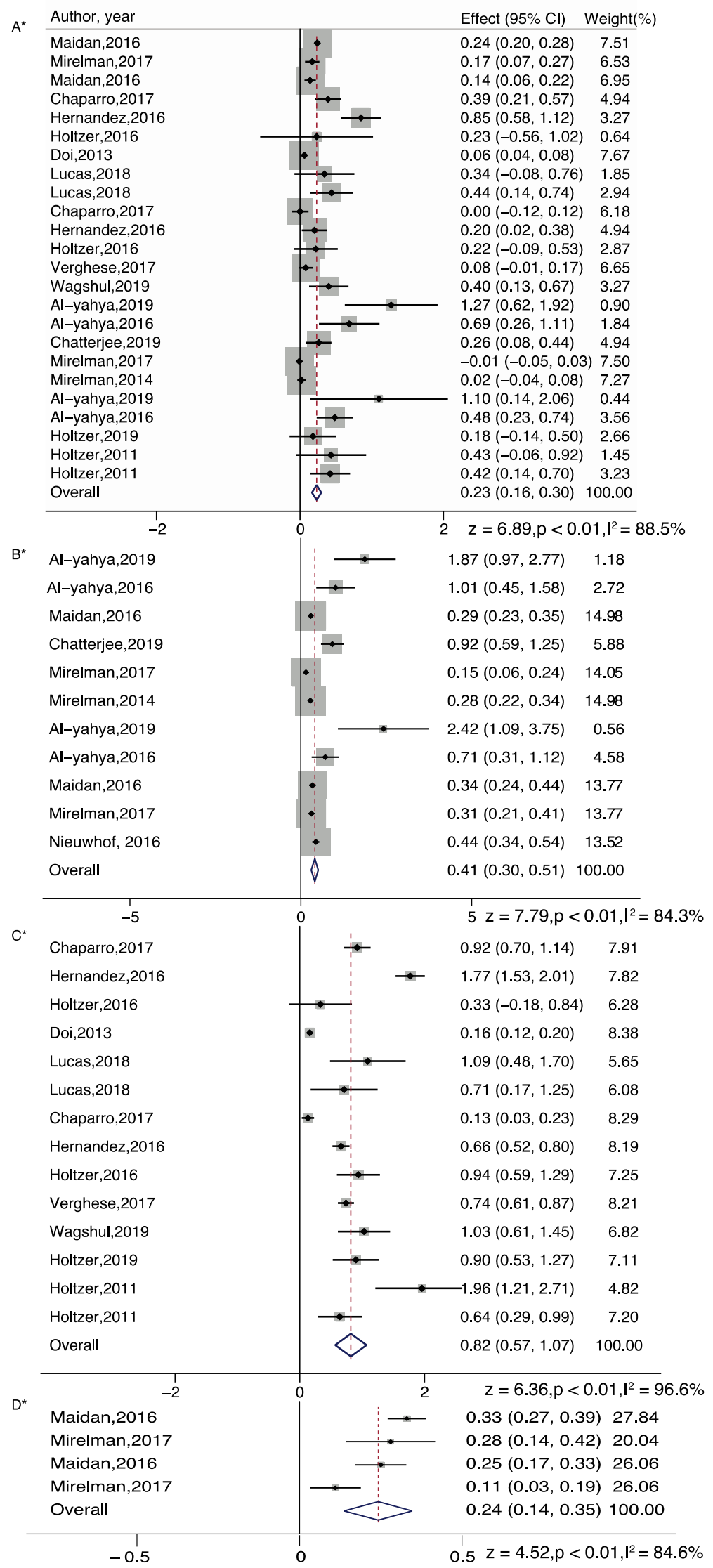

Figure 2. Brain activation differences while doing single task walking (A), serial subtraction task (B), letter generation task (C), or obstacle walking task (D). Note: * represents Random plot metaanalysis [29-31,35-37,42,44,47,48,50,52,55,56,58,62,63]. 


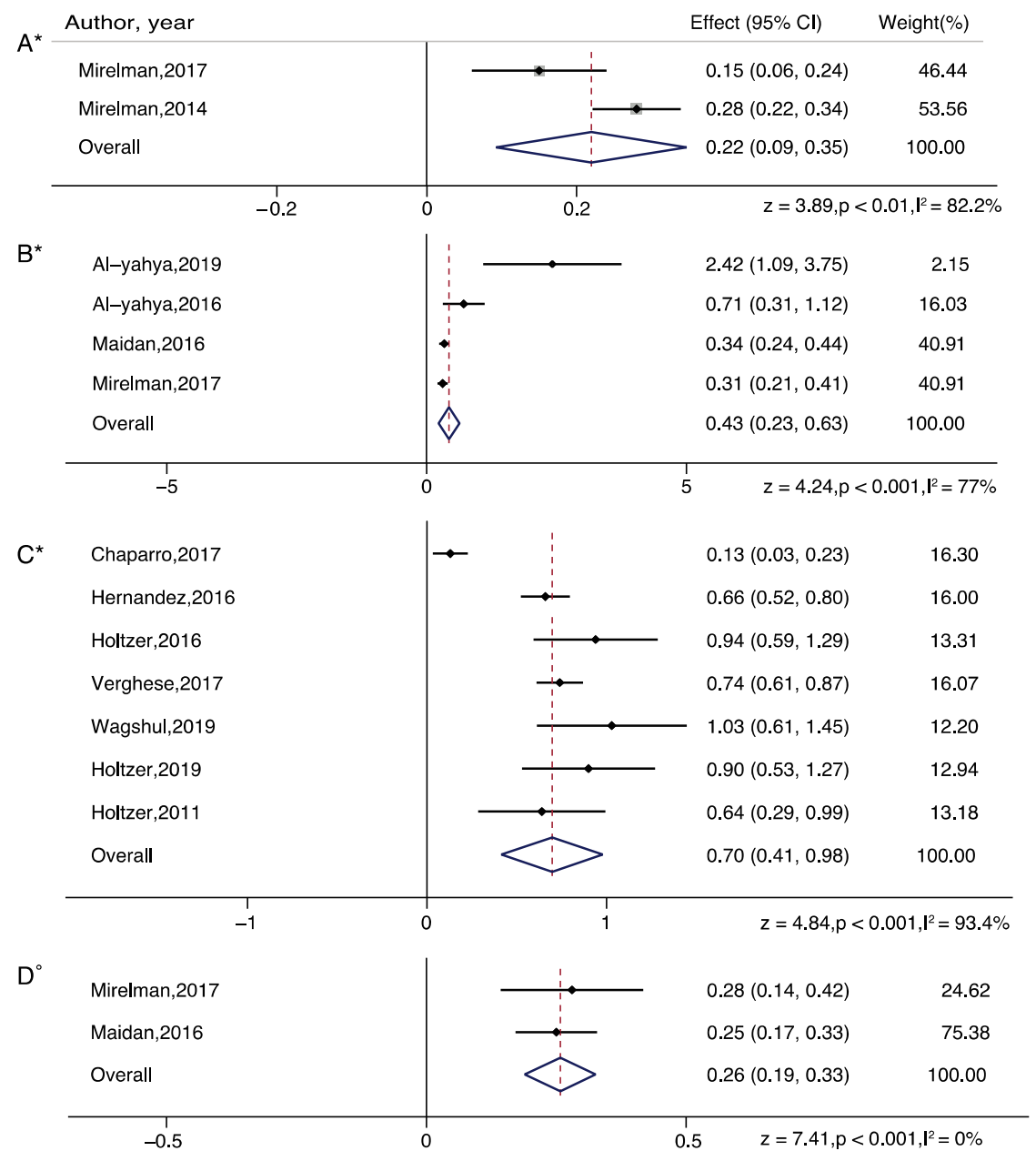

Figure 3. Brain activation differences while doing serial subtraction tasks in healthy young adults (A), and in serial subtraction tasks (B), letter generation tasks (C), or obstacle walking tasks (D) in healthy older adults. Note: ${ }^{\circ}$ represents Fixed plot meta-analysis, ${ }^{*}$ represents Random plot meta-analysis [29-31,35,36,44,47,48,52,55,56,62].

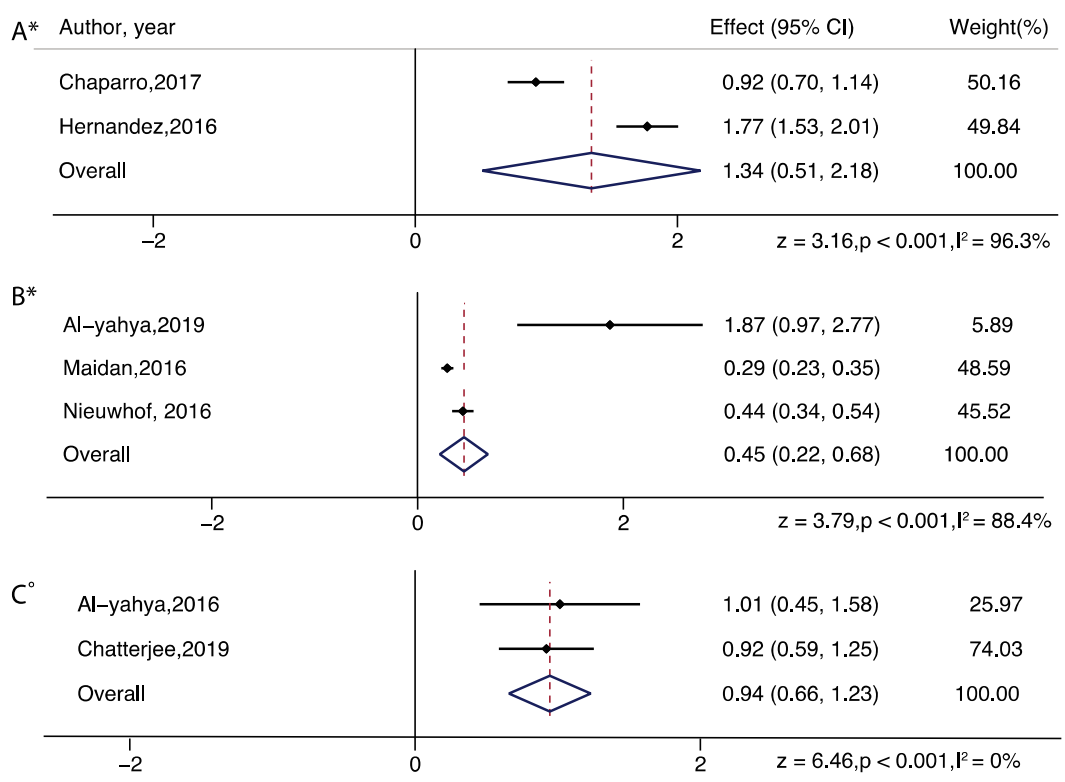

Figure 4. Brain activation differences while doing letter generation task (A) and serial subtraction task $(\mathbf{B}, \mathbf{C})$ in older adults with neurological diseases (multiple sclerosis-A, Parkinson's disease-B, stroke-C). Note: ${ }^{\circ}$ represents Fixed plot meta-analysis, ${ }^{*}$ represents Random plot meta-analysis $[29,30,44,50,52,62,63]$. 
$A^{*}$

\begin{tabular}{|c|c|c|c|}
\hline Author, year & & Effect $(95 \% \mathrm{Cl})$ & Weight(\%) \\
\hline Mirelman,2017 & 1 & $0.16(0.06,0.26)$ & 46.30 \\
\hline Mirelman,2014 & & $0.26(0.18,0.34)$ & 53.70 \\
\hline Overall & & $0.21(0.12,0.31)$ & 100.00 \\
\hline
\end{tabular}

$B^{\circ}$

\begin{tabular}{|c|c|c|c|}
\hline Mirelman,2017 & $\leftarrow$ & $0.14(-0.00,0.28)$ & 42.56 \\
\hline Al-yahya,2019 & 1 & $1.32(-0.32,2.96)$ & 0.31 \\
\hline Maidan,2016 & $\rightarrow$ & $0.20(0.07,0.33)$ & 53.41 \\
\hline Al-yahya,2016 & $\rightarrow$ & $0.23(-0.25,0.71)$ & 3.72 \\
\hline Overall & \rangle & $0.18(0.09,0.27)$ & 100.00 \\
\hline
\end{tabular}

$\mathrm{C}^{*}$

Chaparro,2017

Hernandez,2016

Holtzer,2016

Verghese,2017

Wagshul,2019

Holtzer,2019

Holtzer,2011

Overall

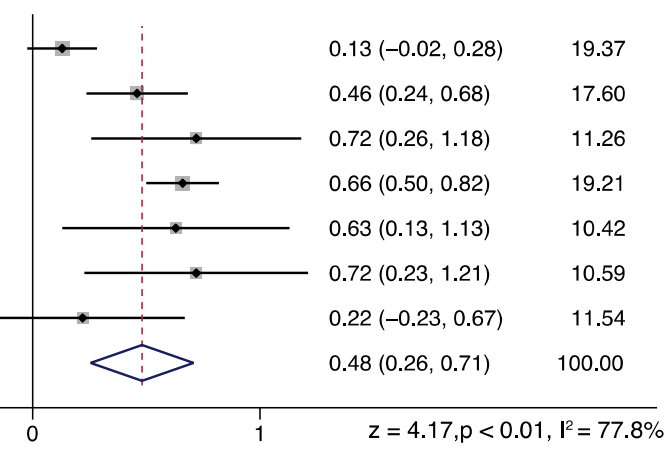

$D^{\circ}$

Mirelman,2017
Maidan,2016
Overall

$-1$

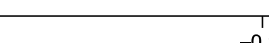

$-0.2$

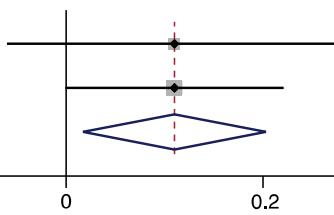

$\begin{array}{lr}0.11(-0.06,0.28) & 29.78 \\ 0.11(-0.00,0.22) & 70.22 \\ 0.11(0.02,0.20) & 100.00 \\ z=2.32, p=0.02, \mathrm{I}^{2}=0 \%\end{array}$

Figure 5. Dual task brain activation differences while doing serial subtraction tasks in healthy young adults (A), and in serial subtraction tasks (B), letter generation tasks (C), and obstacle walking tasks (D) in healthy older adults. Note: ${ }^{\circ}$ represents Fixed plot meta-analysis, ${ }^{*}$ represents Random plot meta-analysis [29-31,35,36,44,47,48,52,62].

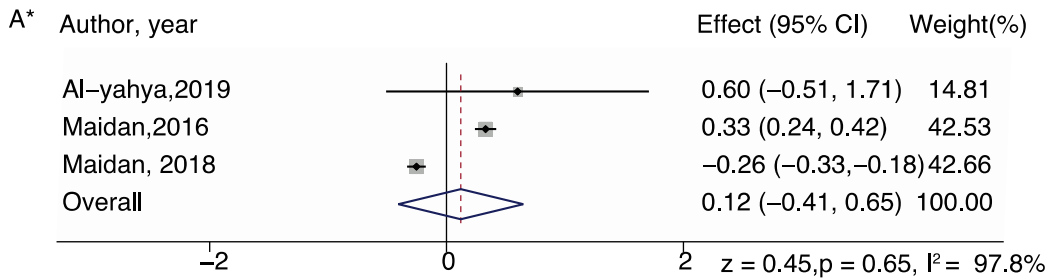

$B^{\circ}$

\begin{tabular}{|c|c|c|}
\hline Al-yahya,2016 & & $0.33(-0.37,1.03) 22.34$ \\
\hline Chatterjee,2019 & • & $0.66(0.28,1.04) \quad 77.66$ \\
\hline Overall & $>$ & $0.59(0.25,0.92) 100.00$ \\
\hline
\end{tabular}

$\mathrm{C}^{*}$

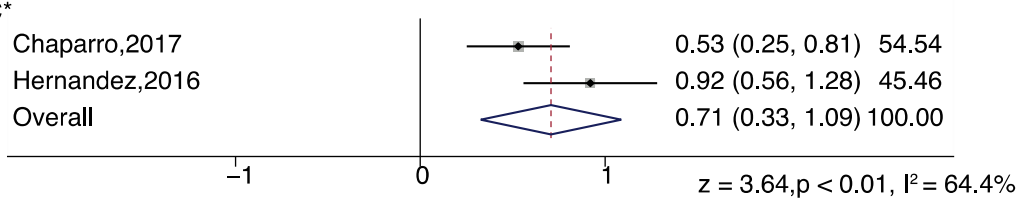

Figure 6. Dual task brain activation differences while doing serial subtraction (Parkinson's disease-(A) and stroke-(B)), letter generation tasks (multiple sclerosis-(C)) in adults with neurological diseases. Note: ${ }^{\circ}$ represents Fixed plot meta-analysis, * represents Random plot meta-analysis [29,30,43,44,52,62,63]. 

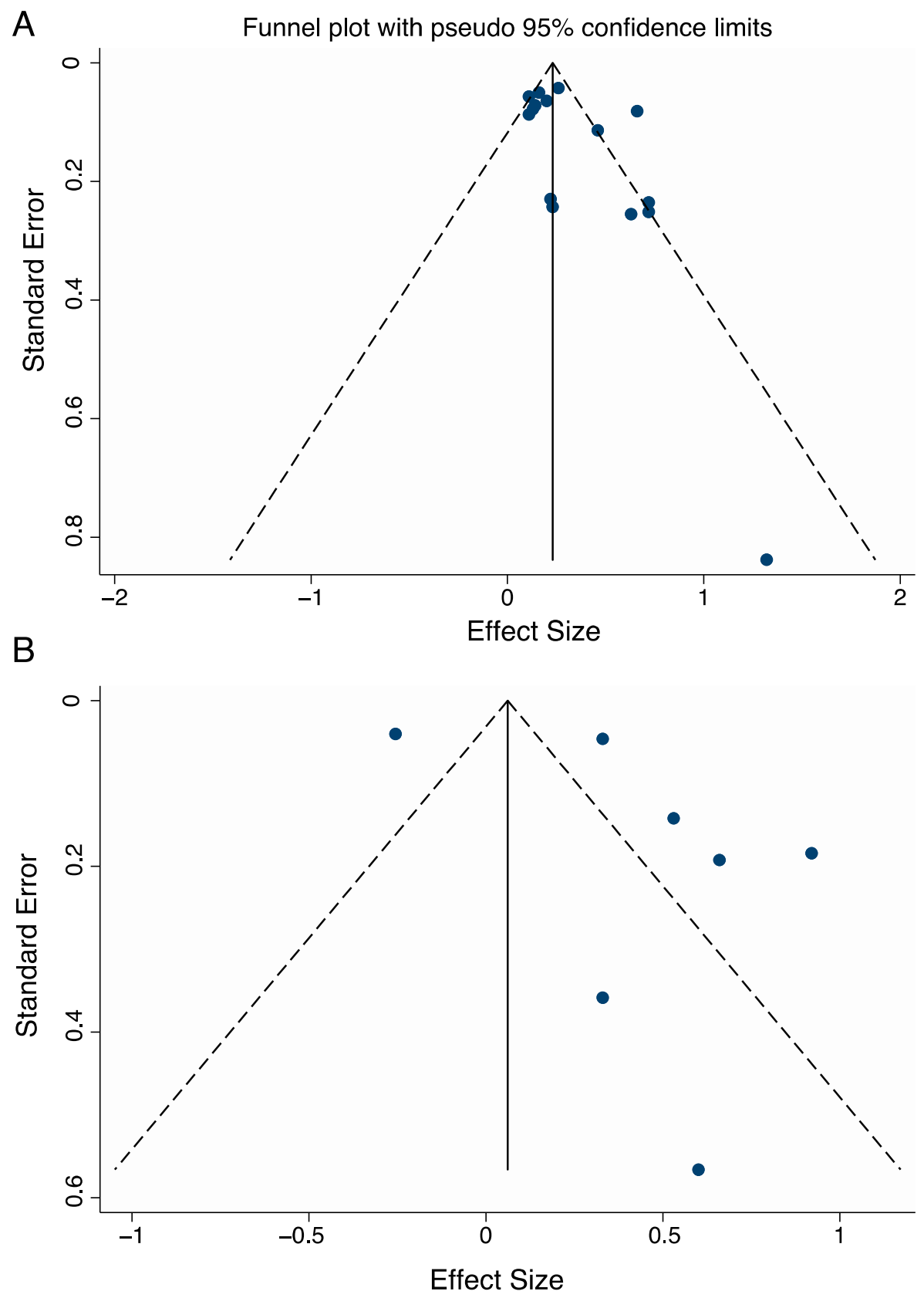

Figure 7. Funnel plots used to examine publication bias in dual task—single task differences in PFC activation in healthy adults (A) and in adults with neurological diseases (B).

\subsection{Study Quality Assessment}

Table 3 reports results of our study quality assessment. Studies included in the review on average scored 16.26 out of 20 and ranged between 10 and 18. The distribution of qualification differed substantially across criteria. Thirty-four out of the 35 studies included in the review clearly described their study population except one study [45]. Six studies out of 35 failed to specify and apply inclusion and exclusion criteria to all participants [38, $40,41,45,46,48]$. Further, 12 studies didn't provide sample size justification $[30,38,40,41,43-$ $45,49,51,52,54,61]$. In term of analysis or paper, exposure of interest was not measured prior to outcome measure in most of the studies except one study [43], also one study did not define their outcome measures completely [30]. Last, the effect of cofounding variables was not measured and adjusted statistically in 12 studies [29,30,39-41,45,49,51,58,59,61,63]. 
Table 3. Study Quality Assessment.

\begin{tabular}{|c|c|c|}
\hline No. & Questions & Score \\
\hline 1 & Was the research question or objective of the study clearly stated? & 2 \\
\hline 2 & Was the study population clearly specified and defined? & 1.94 \\
\hline 3 & $\begin{array}{l}\text { Were inclusion and exclusion criteria for being in the study prespecified and } \\
\text { uniformly applied to all participants? }\end{array}$ & 1.66 \\
\hline 4 & $\begin{array}{c}\text { Was sample size justification, power description or variance and effect } \\
\text { estimates provided? }\end{array}$ & 1.32 \\
\hline 5 & $\begin{array}{l}\text { For analysis of paper, were the exposure(s) of interest measured prior to the } \\
\text { outcome(s) being measured? }\end{array}$ & 0.06 \\
\hline 6 & $\begin{array}{l}\text { Were the exposure measures (independent variables) clearly defined, valid, } \\
\text { reliable, and implemented consistently across all study participants? }\end{array}$ & 2 \\
\hline 7 & $\begin{array}{l}\text { Were the outcome measures (dependent variables) clearly defined, valid, } \\
\text { reliable, and implemented consistently across all study participants? }\end{array}$ & 1.97 \\
\hline 8 & $\begin{array}{l}\text { Were potential confounding variables measured and adjusted statistically for } \\
\text { their impact on the relationship between exposure(s) and outcome(s)? }\end{array}$ & 1.32 \\
\hline 9 & Was dual task clearly defined and uniformly applied to all participants? & 2 \\
\hline \multirow[t]{3}{*}{10} & $\begin{array}{l}\text { Was functional near infrared spectroscopy applied to prefrontal cortex part } \\
\text { of brain and clearly defined in text? }\end{array}$ & 2 \\
\hline & Total & 16.26 \\
\hline & SD & 0.59 \\
\hline
\end{tabular}

\section{Discussion}

This study systematically reviewed and quantitatively synthesized existing scientific evidence on the differences in brain activation during walking while performing cognitive tasks among healthy young adults, healthy older adults, people with Parkinson's disease, stroke and multiple sclerosis. PFC activation of adults with and without neurological disease participated in 35 studies was examined. This systematic review explicitly targeted: (1) quantifying the changes in cortical activation patterns between different dual tasks; (2) quantifying activation differences among different populations; (3) evaluating each study based on the quality assessment criteria. Overall, we show that the performance of obstacle walking, serial subtraction and letter generation tasks while walking result in significant increases in brain activation in the prefrontal cortex relative to standing or walking baselines in adults with and without neurological conditions. Consistent with previous work [24], our meta-analysis showed that letter generation tasks have the largest brain activation effect sizes relative to walking, and that significant differences between dual task and single task gait is seen in persons with stroke, using serial subtraction tasks, and in persons with multiple sclerosis while using letter generation tasks. Furthermore, we found that even normal walking can show significant increases in PFC activation among healthy older adults, people with stroke, and persons with multiple sclerosis.

In terms of aging effects on dual task walking, results were found to differ depending on the baseline used. Larger effect sizes were observed in healthy older adults relative to healthy young adults in serial subtraction tasks while walking, relative to standing baselines (Figure 3), but larger effect sizes were observed in healthy young adults relative to older adults when using single task walking as a baseline (Figure 5). These findings suggest that normal walking may require additional attentional resources in healthy older adults, relative to young adults. These findings are consistent with studies examining agerelated changes while dual task walking, suggesting no differences between healthy young and older adults while performing a visual check task [41], increases in older adults, relative to younger adults, while performing obstacle navigation [47], or decreases in older adults, relative to younger adults, while performing a letter generation task [35]. The discrepancy in results may arise from the effective lateralization in young adults, and utilization of additional cognitive resources to maintain gait performance in older adults, which is in accordance with the CRUNCH (Compensation-Related Utilization of Neural Circuits Hypothesis) model [64]. The CRUNCH model states that at low loads of cognitive demand, 
older adults recruit more cortical regions in comparison to young adults who demonstrate more focal activation based on the task. Thus, differences in concurrent task difficulty and the nature of the task may explain the discrepancy in age-related PFC activation changes while walking and could benefit from additional fNIRS studies with wider spatial coverage and varying difficulty levels to further our understanding of age-related changes.

Consistent with prior findings $[24,36,58]$, we found that the performance of obstacle walking, serial subtraction and letter generation tasks while walking result in significant increases in brain activation in the prefrontal cortex relative to standing or walking baselines in adults with neurological conditions. Significant differences between dual task and single task gait are seen in persons with stroke while using serial subtraction tasks and in persons with multiple sclerosis while using letter generation tasks, even after significant increases in PFC activation while normal walking in both of these populations. The increases in PFC activation observed across adults with neurological conditions is consistent with increased inefficiency in PFC recruitment [65-67] or decreased automaticity of control in walking conditions in neurological populations [68].

Serial subtraction tasks while walking was found to have the largest effect sizes of PFC activation, relative to a standing baseline, consistent with observed brain activation increases of bilateral prefrontal areas in young healthy adults while performing serial subtraction tasks using functional magnetic resonance imaging [69]. Furthermore, relative to walking baselines, letter generation tasks, such walking while reciting alternate letters of the alphabet, were found to have the largest effect sizes of PFC activation, which is suggestive of compensatory cortical activation strategies in adults with neurological conditions, as reduced grey matter volumes in the PFC have been associated with greater increases in overall PFC activation from single task to dual task walking [56]. Thus, as different verbal tasks have distinct structural and functional brain correlates, and fluency tasks such as letter and category fluency have shared distinct neural correlates, small differences between tasks may allow for further clarification on the cortical structures most crucial for controlling gait in complex environments. Differences in PFC activation levels across the neurological populations also raises an important point of differences in cognitive function among these adults. Increases in PFC activation in a difficult working memory task such as serial subtraction task could be originating from prioritization of the task relevant areas as a consequence of further limited resources present in the brain [70]. Furthermore, other areas of cortical activation may be recruited for the support of dual task walking, depending on the cognitive task involved while walking and the specific neurological condition. Thus, care should be taken to utilize specific dual task walking paradigms whose activation can be captured by a given region of interest with fNIRS.

\subsection{Clinical Implications}

Dual task walking paradigms may better approximate locomotion demands in natural settings where individuals are required to negotiate visual and auditory stimuli that interfere with the maintenance of safe gait. The ecological validity of such paradigms may thus confer improved predictive utility. For example, in older adults, worse dual task walking performance was associated with increased risk of incident frailty, disability and mortality even when adjusting for single task walking [71]. Poor dual-task walking is also predictive of falls in aging and neurological populations [72]. Specifically, with respect to fNIRS-derived $\mathrm{HbO}_{2}$ in the PFC, as assessed during walking, higher activation during dual but not single task conditions predicted increased risk of incident falls [55]. This finding suggested that over activation of the PFC during cognitively demanding walking was indicative of inefficient utilization of brain resources that predisposed individuals to greater falls risk. Establishing robust and reliable effect sizes for fNIRS-derived $\mathrm{HbO}_{2}$ during active walking in normal and disease populations is critical to determine its potential clinical utility as a tool for risk assessment of mobility-related decline and disability outcomes. Furthermore, within session training in dual-task walking resulted in improved performance and reduced fNIRS-derived $\mathrm{HbO}_{2}$ in the PFC (i.e., improved 
neural efficiency) in older adults [31]. This improvement in PFC efficiency was due to within session training in dual-task walking, however, was moderated by the presence of mild cognitive impairments [73] and fear of falling [32], an important risk factor for falls and other mobility-related outcomes [74-78]. The utility of fNIRS-derived $\mathrm{HbO}_{2}$, assessed during dual-task walking, as primary or secondary outcomes in clinical trials will have to be established in future research. fNIRS-derived measures may help ensure that dosage of any exercise prescription is comparable across individuals [79]. One limitation of current research is concerned with the variety of dual-task paradigms and experimental procedures used in different studies, as seen in other reviews [80]. While the robust effects sizes reported in the present study are encouraging standardization of tasks and procedures would be a necessary step moving forward. It is noteworthy that such efforts exist not only in traditional self-report, behavioral and cognitive outcomes in clinical research but also in recent neuroimaging outcomes [24]. A recent study revealed that using different filters and processing algorithms yielded some differences in the extracted values of $\mathrm{HbO}_{2}$ and $\mathrm{Hb}$ values of walking under single and dual talking conditions though the task effect remained similar [81]. Establishing transparent consensus criteria for fNIRS data processing, specifically with respect to dual task walking paradigms, will also be necessary for efforts to determine and enhance its utility in clinical research, as demonstrated by recent attempts to establish best practices [82,83].

\subsection{Other Implications of fNIRS}

Besides use of fNIRS in dual task paradigms, it is also used in community navigation tasks with augmented reality [84] or during environmentally complex tasks [85] in healthy young adults. This is due to the ability of fNIRS to work as a practical mobile neuroimaging device in complex real-world environments.

Measuring brain activity during natural environmental settings is a growing field and use of fNIRS has served as an advantageous tool, especially, in the area of Neuroergonomics, in which one can implement natural work settings and measure the brain activity of the individual while wearing fNIRS systems [86,87]. Lastly, while using fNIRS, researchers need to be aware of its application in different populations and in different experimental paradigms. Although, the focus of this systematic review was dual task paradigms, fNIRS has a wide range of potential applications.

\subsection{Limitations}

We found changes in the PFC activation among adults with and without neurological diseases, however, few studies have reported both $\mathrm{HbO} 2$ and $\mathrm{Hb}$ values, and the number of studies in each subgroup meta-analysis varied which may have led to biased findings. Although no publication bias was detected using Egger's test in clinical populations nor in healthy adults, the small number of studies limits the power of the test to detect bias. The results of these studies should be interpreted with caution, as funnel plots suggest the presence of publication bias for the serial subtraction and letter generation dual tasks in patient populations but not in healthy older adults. We need more studies with these tasks to confirm our subgroup analysis. Given the limited number of studies, we were unable to carry out sub-group analyses on influencing factors such as overground versus treadmill walking, dual task prioritization, and processing of systemic confounders on PFC activation. Future work should examine the effect of these factors on PFC activation among adults with and without neurological diseases.

\section{Conclusions}

The current review and meta-analytic study provide comprehensive information on PFC activation differences measured by fNIRS and yields novel information on the significance of cognitive tasks to be used while walking in adults with different neurological conditions. fNIRS technology seems to be a promising tool to shed light on the functioning of cortical areas in motor control. The information provided regarding the 
robustness of fNIRS-derived $\mathrm{HbO}_{2}$ assessed during active walking under single and dualtask conditions is a critical step towards establishing its utility as a risk factor of adverse cognitive and mobility outcomes in normal and disease populations. This information is also critical for establishing the potential utility of fNIRS-derived $\mathrm{HbO}_{2}$ as a treatment outcome measure that may enhance the monitoring and evaluation of the effectiveness of intervention programs among healthy adults and those with different neurological and non-neurological conditions.

Author Contributions: Conceptualization, A.B. and M.E.H.; methodology, A.B. and M.E.H.; formal analysis, A.B. and M.E.H.; funding acquisition, R.H. All authors have read and agreed to the published version of the manuscript.

Funding: This research was funded by the National Institutes of Health, grant number R01NS109023.

Institutional Review Board Statement: Not applicable.

Informed Consent Statement: Not applicable.

Data Availability Statement: No new data were created or analyzed in this study. Data sharing is not applicable to this article.

Conflicts of Interest: The authors declare no conflict of interest. The sponsors had no role in the design, execution, interpretation, or writing of the study.

\section{Appendix A}

Search Terms Used for Each Database:

\begin{tabular}{|c|c|}
\hline Database & Search Terms \\
\hline PubMed & 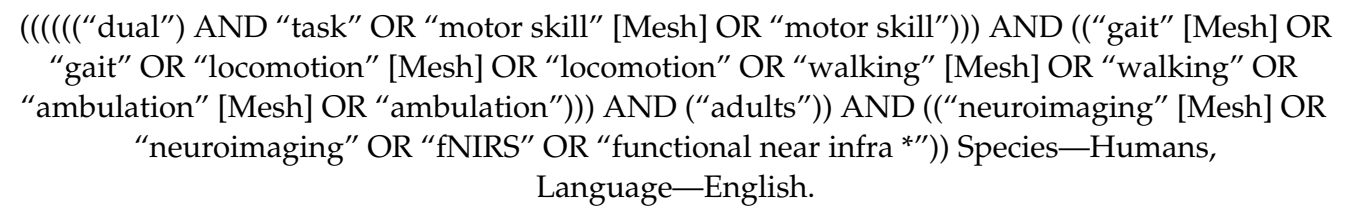 \\
\hline $\begin{array}{c}\text { Cumulative Index of Nursing } \\
\text { and Allied Health }\end{array}$ & $\begin{array}{c}\text { ((“dual task") AND (“walking” OR “gait” OR “locomotion” OR “ambulation") AND (“adults") } \\
\text { AND ("neuroimaging" OR "fNIRS" OR “functional near infra *”)))Limiters: English language; } \\
\text { Human, Journal article }\end{array}$ \\
\hline Web of Science & 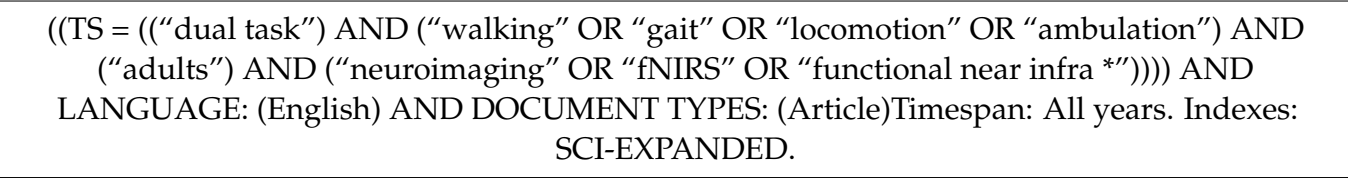 \\
\hline PsycINFO & 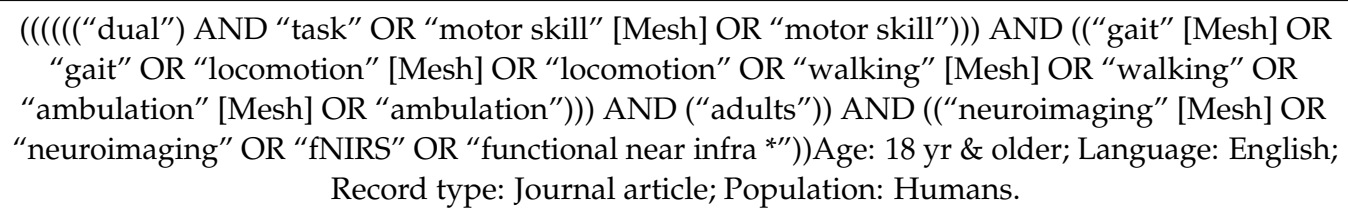 \\
\hline Scopus & 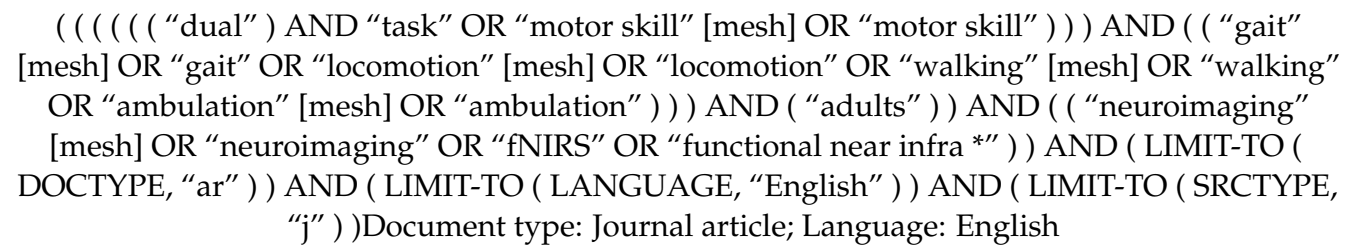 \\
\hline
\end{tabular}

Note: * represents wild card. 


\section{Appendix B}

\begin{tabular}{|c|c|c|c|c|}
\hline Study ID. & Author & $\begin{array}{l}\text { Treadmill or Overground } \\
\text { Walking? }\end{array}$ & Prioritization of Dual Task? & $\begin{array}{l}\text { Additional Noise } \\
\text { Processing? }\end{array}$ \\
\hline 1 & Beurskens 2014 [41] & Treadmill & No & Yes \\
\hline 2 & Chen 2017 [57] & Overground & Pay equal attention & Yes \\
\hline 3 & Fraser 2016 [59] & Treadmill & Pay equal attention & NR \\
\hline 4 & George 2019 [60] & Overground & Pay equal attention & NR \\
\hline 5 & Holtzer 2011 [35] & Overground & Pay equal attention & Yes \\
\hline 6 & Holtzer 2016 [36] & Overground & Pay equal attention & Yes \\
\hline 7 & Holtzer 2016 [37] & Overground & Pay equal attention & Yes \\
\hline 8 & Holtzer 2017 [34] & Overground & Pay equal attention & Yes \\
\hline 9 & Holtzer 2018 [33] & Overground & Pay equal attention & Yes \\
\hline 10 & Holtzer 2019 [31] & Overground & Pay equal attention & Yes \\
\hline 11 & Holtzer 2019 [32] & Overground & Pay equal attention & Yes \\
\hline 12 & Lin 2016 [38] & Overground & No & Yes \\
\hline 13 & Lu 2015 [40] & Overground & Yes & Yes \\
\hline 14 & Lucas 2019 [42] & Overground & Pay equal attention & Yes \\
\hline 15 & Meester 2014 [45] & Treadmill & No & NR \\
\hline 16 & Metzger 2017 [46] & Treadmill & No & NR \\
\hline 17 & Mirelman 2014 [48] & Overground & No & Yes \\
\hline 18 & Mirelman 2017 [47] & Overground & No & Yes \\
\hline 19 & Osofyundiya 2016 [51] & Overground & Pay equal attention & NR \\
\hline 20 & Stuart 2018 [54] & Treadmill & No & Yes \\
\hline 21 & Verghese 2016 [55] & Overground & Pay equal attention & Yes \\
\hline 22 & Wagshul 2019 [56] & Overground & Pay equal attention & Yes \\
\hline 23 & Al-Yahya 2019 [29] & Treadmill & No & Yes \\
\hline 24 & Maidan 2016 [44] & Overground & No & Yes \\
\hline 25 & Maidan 2018 [43] & Treadmill & No & Yes \\
\hline 26 & Nieuwhof 2016 [50] & Overground & NR & NR \\
\hline 27 & Al-Yahya 2016 [30] & Treadmill & No & Yes \\
\hline 28 & Chatterjee 2019 [63] & Overground & No & NR \\
\hline 29 & Hermand 2019 [61] & Overground & Pay equal attention & Yes \\
\hline 30 & Liu 2018 [39] & Overground & No & Yes \\
\hline 31 & Mori 2017 [49] & Overground & No & NR \\
\hline 32 & Chaparro 2017 [52] & Treadmill & No & Yes \\
\hline 33 & Hernandez 2016 [62] & Overground & No & Yes \\
\hline 34 & Saleh 2018 [53] & Overground & Pay equal attention & Yes \\
\hline 35 & Doi 2013 [58] & Overground & No & NR \\
\hline
\end{tabular}

Note: NR = Not reported. 


\section{References}

1. Hamacher, D.; Herold, F.; Wiegel, P.; Hamacher, D.; Schega, L. Brain Activity during Walking: A Systematic Review. Neurosci. Biobehav. Rev. 2015, 57, 310-327. [CrossRef] [PubMed]

2. Caetano, M.J.D.; Menant, J.C.; Schoene, D.; Pelicioni, P.H.S.; Sturnieks, D.L.; Lord, S.R. Sensorimotor and Cognitive Predictors of Impaired Gait Adaptability in Older People. J. Gerontol. Ser. A Biol. Sci. Med. Sci. 2016, 72, glw171. [CrossRef] [PubMed]

3. Montero-Odasso, M.; Verghese, J.; Beauchet, O.; Hausdorff, J.M. Gait and Cognition: A Complementary Approach to Understanding Brain Function and the Risk of Falling. J. Am. Geriatr. Soc. 2012. [CrossRef] [PubMed]

4. Holtzer, R.; Epstein, N.; Mahoney, J.R.; Izzetoglu, M.; Blumen, H.M. Neuroimaging of Mobility in Aging: A Targeted Review. J. Gerontol. Ser. A Biol. Sci. Med. Sci. 2014, 69, 1375-1388. [CrossRef]

5. Cutini, S.; Moro, S.B.; Bisconti, S. Functional near Infrared Optical Imaging in Cognitive Neuroscience: An Introductory Review. J. Near Infrared Spectrosc. 2012, 20, 75-92. [CrossRef]

6. Cutini, S.; Brigadoi, S. Unleashing the Future Potential of Functional Near-Infrared Spectroscopy in Brain Sciences. J. Neurosci. Methods 2014, 232, 152-156. [CrossRef] [PubMed]

7. Bunce, S.C.; Izzetoglu, M.; Izzetoglu, K.; Onaral, B.; Pourrezaei, K. Functional Near-Infrared Spectroscopy. IEEE Eng. Med. Biol. Mag. 2006, 25, 54-62. [CrossRef] [PubMed]

8. Saliba, J.; Bortfeld, H.; Levitin, D.J.; Oghalai, J.S. Functional Near-Infrared Spectroscopy for Neuroimaging in Cochlear Implant Recipients. Hear. Res. 2016, 338, 64-75. [CrossRef] [PubMed]

9. Lloyd-Fox, S.; Blasi, A.; Elwell, C.E. Illuminating the Developing Brain: The Past, Present and Future of Functional near Infrared Spectroscopy. Neurosci. Biobehav. Rev. 2010, 34, 269-284. [CrossRef] [PubMed]

10. Smith, M. Shedding Light on the Adult Brain: A Review of the Clinical Applications of near-Infrared Spectroscopy. Philos. Trans. R. Soc. A Math. Phys. Eng. Sci. 2011, 369, 4452-4469. [CrossRef] [PubMed]

11. Thompson, J.; Sebastianelli, W.; Slobounov, S. EEG and Postural Correlates of Mild Traumatic Brain Injury in Athletes. Neurosci. Lett. 2005, 377, 158-163. [CrossRef]

12. Maki, A.; Yamashita, Y.; Ito, Y.; Watanabe, E.; Mayanagi, Y.; Koizumi, H. Spatial and Temporal Analysis of Human Motor Activity Using Noninvasive NIR Topography. Med. Phys. 1995, 22, 1997-2005. [CrossRef] [PubMed]

13. Watanabe, K.; Funahashi, S. Toward an Understanding of the Neural Mechanisms Underlying Dual-Task Performance: Contribution of Comparative Approaches Using Animal Models. Neurosci. Biobehav. Rev. 2018, 84, 12-28. [CrossRef] [PubMed]

14. Baddeley, A. Exploring the Central Executive. Q. J. Exp. Psychol. Sect. A Hum. Exp. Psychol. 1996. [CrossRef]

15. Just, M.A.; Carpenter, P.A. A Capacity Theory of Comprehension: Individual Differences in Working Memory. Psychol. Rev. 1992. [CrossRef] [PubMed]

16. Holtzer, R.; Wang, C.; Verghese, J. Performance Variance on Walking While Talking Tasks: Theory, Findings, and Clinical Implications. Age 2014, 36, 373-381. [CrossRef] [PubMed]

17. Holtzer, R.; Wang, C.; Verghese, J. The Relationship between Attention and Gait in Aging: Facts and Fallacies. Motor Control 2012, 16, 64-80. [CrossRef] [PubMed]

18. D'esposito, M.; Detre, J.A.; Alsop, D.C.; Shin, R.K.; Scott, A.; Grossman, M. The Neural Basis of the Central Executive System of Working Memory. Nature 1995. [CrossRef] [PubMed]

19. Ferrari, M.; Quaresima, V. A Brief Review on the History of Human Functional Near-Infrared Spectroscopy (FNIRS) Development and Fields of Application. Neuroimage 2012, 63, 921-935. [CrossRef] [PubMed]

20. Kamran, M.A.; Mannan, M.M.N.; Jeong, M.Y. Cortical Signal Analysis and Advances in Functional Near-Infrared Spectroscopy Signal: A Review. Front. Hum. Neurosci. 2016, 10. [CrossRef]

21. Orihuela-Espina, F.; Leff, D.R.; James, D.R.C.; Darzi, A.W.; Yang, G.Z. Quality Control and Assurance in Functional near Infrared Spectroscopy (FNIRS) Experimentation. Phys. Med. Biol. 2010. [CrossRef] [PubMed]

22. Herold, F.; Wiegel, P.; Scholkmann, F.; Thiers, A.; Hamacher, D.; Schega, L. Functional Near-Infrared Spectroscopy in Movement Science: A Systematic Review on Cortical Activity in Postural and Walking Tasks. Neurophotonics 2017. [CrossRef] [PubMed]

23. Vitorio, R.; Stuart, S.; Rochester, L.; Alcock, L.; Pantall, A. FNIRS Response during Walking-Artefact or Cortical Activity? A Systematic Review. Neurosci. Biobehav. Rev. 2017, 83, 160-172. [CrossRef] [PubMed]

24. Pelicioni, P.H.S.S.; Tijsma, M.; Lord, S.R.; Menant, J. Prefrontal Cortical Activation Measured by FNIRS during Walking: Effects of Age, Disease and Secondary Task. Peerj 2019, 7. [CrossRef]

25. Udina, C.; Avtzi, S.; Durduran, T.; Holtzer, R.; Rosso, A.L.; Castellano-Tejedor, C.; Perez, L.M.; Soto-Bagaria, L.; Inzitari, M. Functional Near-Infrared Spectroscopy to Study Cerebral Hemodynamics in Older Adults During Cognitive and Motor Tasks: A Review. Front. Aging Neurosci. 2020. [CrossRef]

26. Moher, D.; Shamseer, L.; Clarke, M.; Ghersi, D.; Liberatî, A.; Petticrew, M.; Shekelle, P.; Stewart, L.A.; Group, P.-P. Preferred Reporting Items for Systematic Review and Meta-Analysis Protocols (PRISMA-P) 2015 Statement. Syst. Rev. 2015, 4, 1-9. [CrossRef]

27. Littell, J.H.; Corcoran, J.; Pillai, V.K. Systematic Reviews and Meta-Analysis; Oxford University Press: New York, NY, USA, 2008.

28. Allison, J.J. Quality Assessment Tools. Med. Care 2003. [CrossRef] [PubMed]

29. Al-Yahya, E.; Mahmoud, W.; Meester, D.; Esser, P.; Dawes, H. Neural Substrates of Cognitive Motor Interference during Walking; Peripheral and Central Mechanisms. Front. Hum. Neurosci. 2019, 12. [CrossRef] [PubMed] 
30. Al-Yahya, E.; Johansen-Berg, H.; Kischka, U.; Zarei, M.; Cockburn, J.; Dawes, H. Prefrontal Cortex Activation While Walking Under Dual-Task Conditions in Stroke: A Multimodal Imaging Study. Neurorehabil. Neural Repair 2016, 30, 591-599. [CrossRef] [PubMed]

31. Holtzer, R.; Izzetoglu, M.; Chen, M.; Wang, C. Distinct FNIRS-Derived HbO2 Trajectories during the Course and over Repeated Walking Trials under Single-and Dual-Task Conditions: Implications for Within Session Learning and Prefrontal Cortex Efficiency in Older Adults. J. Gerontol. Ser. A Biol. Sci. Med. Sci. 2019, 74, 1076-1083. [CrossRef]

32. Holtzer, R.; Kraut, R.; Izzetoglu, M.; Ye, K. The Effect of Fear of Falling on Prefrontal Cortex Activation and Efficiency during Walking in Older Adults. Geroscience 2019, 41, 89-100. [CrossRef] [PubMed]

33. Holtzer, R.; George, C.J.; Izzetoglu, M.; Wang, C. The Effect of Diabetes on Prefrontal Cortex Activation Patterns during Active Walking in Older Adults. Brain Cogn. 2018, 125, 14-22. [CrossRef] [PubMed]

34. Holtzer, R.; Schoen, C.; Demetriou, E.; Mahoney, J.R.; Izzetoglu, M.; Wang, C.; Verghese, J. Stress and Gender Effects on Prefrontal Cortex Oxygenation Levels Assessed during Single and Dual-Task Walking Conditions. Eur. J. Neurosci. 2017, 45, 660-670. [CrossRef]

35. Holtzer, R.; Mahoney, J.R.; Izzetoglu, M.; Izzetoglu, K.; Onaral, B.; Verghese, J. FNIRS Study of Walking and Walking While Talking in Young and Old Individuals. J. Gerontol. Ser. A Biol. Sci. Med. Sci. 2011, 66, 879-887. [CrossRef] [PubMed]

36. Holtzer, R.; Verghese, J.; Allali, G.; Izzetoglu, M.; Wang, C.; Mahoney, J.R. Neurological Gait Abnormalities Moderate the Functional Brain Signature of the Posture First Hypothesis. Brain Topogr. 2016, 29, 334-343. [CrossRef]

37. Holtzer, R.; Yuan, J.; Verghese, J.; Mahoney, J.R.; Izzetoglu, M.; Wang, C. Interactions of Subjective and Objective Measures of Fatigue Defined in the Context of Brain Control of Locomotion. J. Gerontol. Ser. A Biol. Sci. Med. Sci. 2016, 72, 417-423. [CrossRef] [PubMed]

38. Lin, M.-I.B.; Lin, K.-H. Walking While Performing Working Memory Tasks Changes the Prefrontal Cortex Hemodynamic Activations and Gait Kinematics. Front. Behav. Neurosci. 2016, 10. [CrossRef] [PubMed]

39. Liu, Y.-C.C.; Yang, Y.-R.R.; Tsai, Y.-A.A.; Wang, R.-Y.Y.; Lu, C.-F.F. Brain Activation and Gait Alteration during Cognitive and Motor Dual Task Walking in Stroke-A Functional Near-Infrared Spectroscopy Study. IEEE Trans. Neural Syst. Rehabil. Eng. 2018, 26, 2416-2423. [CrossRef] [PubMed]

40. Lu, C.F.; Liu, Y.C.; Yang, Y.R.; Wu, Y.T.; Wang, R.Y. Maintaining Gait Performance by Cortical Activation during Dual-Task Interference: A Functional near-Infrared Spectroscopy Study. PLoS ONE 2015, 10. [CrossRef] [PubMed]

41. Beurskens, R.; Helmich, I.; Rein, R.; Bock, O. Age-Related Changes in Prefrontal Activity during Walking in Dual-Task Situations: A FNIRS Study. Int. J. Psychophysiol. 2014, 92, 122-128. [CrossRef] [PubMed]

42. Lucas, M.; Wagshul, M.E.; Izzetoglu, M.; Holtzer, R. Moderating Effect of White Matter Integrity on Brain Activation during Dual-Task Walking in Older Adults. Journals Gerontol. Ser. A Biol. Sci. Med. Sci. 2019, 74, 435-441. [CrossRef] [PubMed]

43. Maidan, I.; Nieuwhof, F.; Bernad-Elazari, H.; Bloem, B.R.; Giladi, N.; Hausdorff, J.M.; Claassen, J.A.H.R.; Mirelman, A. Evidence for Differential Effects of 2 Forms of Exercise on Prefrontal Plasticity During Walking in Parkinson's Disease. Neurorehabil. Neural Repair 2018, 32, 200-208. [CrossRef]

44. Maidan, I.; Nieuwhof, F.; Bernad-Elazari, H.; Reelick, M.F.; Bloem, B.R.; Giladi, N.; Deutsch, J.E.; Hausdorff, J.M.; Claassen, J.A.H.; Mirelman, A. The Role of the Frontal Lobe in Complex Walking among Patients With Parkinson's Disease and Healthy Older Adults: An FNIRS Study. Neurorehabil. Neural Repair 2016, 30, 963-971. [CrossRef] [PubMed]

45. Meester, D.; Al-Yahya, E.; Dawes, H.; Martin-Fagg, P.; Pinon, C. Associations between Prefrontal Cortex Activation and H-Reflex Modulation during Dual Task Gait. Front. Hum. Neurosci. 2014, 8. [CrossRef] [PubMed]

46. Metzger, F.G.; Ehlis, A.-C.; Haeussinger, F.B.; Schneeweiss, P.; Hudak, J.; Fallgatter, A.J.; Schneider, S. Functional brain imaging of walking while talking-An fnirs study. Neuroscience 2017, 343, 85-93. [CrossRef] [PubMed]

47. Mirelman, A.; Maidan, I.; Bernad-Elazari, H.; Shustack, S.; Giladi, N.; Hausdorff, J.M. Effects of Aging on Prefrontal Brain Activation during Challenging Walking Conditions. Brain Cogn. 2017, 115, 41-46. [CrossRef]

48. Mirelman, A.; Maidan, I.; Bernad-Elazari, H.; Nieuwhof, F.; Reelick, M.; Giladi, N.; Hausdorff, J.M. Increased Frontal Brain Activation during Walking While Dual Tasking: An FNIRS Study in Healthy Young Adults. J. Neuroeng. Rehabil. 2014, 11. [CrossRef] [PubMed]

49. Mori, T.; Takeuchi, N.; Izumi, S.I. Prefrontal Cortex Activation during a Dual Task in Patients with Stroke. Gait Posture 2018, 59, 193-198. [CrossRef] [PubMed]

50. Nieuwhof, F.; Reelick, M.F.; Maidan, I.; Mirelman, A.; Hausdorff, J.M.; Olde Rikkert, M.G.M.; Bloem, B.R.; Muthalib, M.; Claassen, J.A.H.R. Measuring Prefrontal Cortical Activity during Dual Task Walking in Patients with Parkinson's Disease: Feasibility of Using a New Portable FNIRS Device. Pilot Feasibility Stud. 2016, 2, 1-11. [CrossRef] [PubMed]

51. Osofundiya, O.; Benden, M.E.; Dowdy, D.; Mehta, R.K. Obesity-Specific Neural Cost of Maintaining Gait Performance under Complex Conditions in Community-Dwelling Older Adults. Clin. Biomech. 2016, 35, 42-48. [CrossRef] [PubMed]

52. Chaparro, G.; Balto, J.M.; Sandroff, B.M.; Holtzer, R.; Izzetoglu, M.; Motl, R.W.; Hernandez, M.E. Frontal Brain Activation Changes Due to Dual-Tasking under Partial Body Weight Support Conditions in Older Adults with Multiple Sclerosis. J. Neuroeng. Rehabil. 2017, 14. [CrossRef] [PubMed]

53. Saleh, S.; Sandroff, B.M.; Vitiello, T.; Owoeye, O.; Hoxha, A.; Hake, P.; Goverover, Y.; Wylie, G.; Yue, G.; DeLuca, J. The Role of Premotor Areas in Dual Tasking in Healthy Controls and Persons with Multiple Sclerosis: An FNIRS Imaging Study. Front. Behav. Neurosci. 2018, 12, 1-11. [CrossRef] [PubMed] 
54. Stuart, S.; Alcock, L.; Rochester, L.; Vitorio, R.; Pantall, A. Monitoring Multiple Cortical Regions during Walking in Young and Older Adults: Dual-Task Response and Comparison Challenges. Int. J. Psychophysiol. 2019, 135, 63-72. [CrossRef] [PubMed]

55. Verghese, J.; Wang, C.; Ayers, E.; Izzetoglu, M.; Holtzer, R. Brain Activation in High-Functioning Older Adults and Falls: Prospective Cohort Study. Neurology 2017, 88, 191-197. [CrossRef]

56. Wagshul, M.E.; Lucas, M.; Ye, K.; Izzetoglu, M.; Holtzer, R. Multi-Modal Neuroimaging of Dual-Task Walking: Structural MRI and FNIRS Analysis Reveals Prefrontal Grey Matter Volume Moderation of Brain Activation in Older Adults. Neuroimage 2019, 189, 745-754. [CrossRef]

57. Chen, M.; Pillemer, S.; England, S.; Izzetoglu, M.; Mahoney, J.R.; Holtzer, R. Neural Correlates of Obstacle Negotiation in Older Adults: An FNIRS Study. Gait Posture 2017, 58, 130-135. [CrossRef] [PubMed]

58. Doi, T.; Makizako, H.; Shimada, H.; Park, H.; Tsutsumimoto, K.; Uemura, K.; Suzuki, T. Brain Activation during Dual-Task Walking and Executive Function among Older Adults with Mild Cognitive Impairment: A FNIRS Study. Aging Clin. Exp. Res. 2013, 25, 539-544. [CrossRef] [PubMed]

59. Fraser, S.A.; Dupuy, O.; Pouliot, P.; Lesage, F.; Bherer, L. Comparable Cerebral Oxygenation Patterns in Younger and Older Adults during Dual-Task Walking with Increasing Load. Front. Aging Neurosci. 2016, 8. [CrossRef] [PubMed]

60. George, C.J.; Verghese, J.; Izzetoglu, M.; Wang, C.; Holtzer, R. The Effect of Polypharmacy on Prefrontal Cortex Activation during Single and Dual Task Walking in Community Dwelling Older Adults. Pharmacol. Res. 2019, 139, 113-119. [CrossRef] [PubMed]

61. Hermand, E.; Tapie, B.; Dupuy, O.; Fraser, S.; Compagnat, M.; Salle, J.Y.; Daviet, J.C.; Perrochon, A. Prefrontal Cortex Activation during Dual Task with Increasing Cognitive Load in Subacute Stroke Patients: A Pilot Study. Front. Aging Neurosci. 2019, 10, 1-6. [CrossRef]

62. Hernandez, M.E.; Holtzer, R.; Chaparro, G.; Jean, K.; Balto, J.M.; Sandroff, B.M.; Izzetoglu, M.; Motl, R.W. Brain Activation Changes during Locomotion in Middle-Aged to Older Adults with Multiple Sclerosis. J. Neurol. Sci. 2016, 370, 277-283. [CrossRef]

63. Chatterjee, S.A.; Fox, E.J.; Daly, J.J.; Rose, D.K.; Wu, S.S.; Christou, E.A.; Hawkins, K.A.; Otzel, D.M.; Butera, K.A.; Skinner, J.W.; et al. Interpreting Prefrontal Recruitment During Walking After Stroke: Influence of Individual Differences in Mobility and Cognitive Function. Front. Hum. Neurosci. 2019, 13. [CrossRef]

64. Reuter-Lorenz, P.A.; Cappell, K.A. Neurocognitive Aging and the Compensation Hypothesis. Curr. Dir. Psychol. Sci. 2008. [CrossRef]

65. Audoin, B.; Van Au Duong, M.; Ranjeva, J.P.; Ibarrola, D.; Malikova, I.; Confort-Gouny, S.; Soulier, E.; Viout, P.; Ali-Chérif, A.; Pelletier, J.; et al. Magnetic Resonance Study of the Influence of Tissue Damage and Cortical Reorganization on PASAT Performance at the Earliest Stage of Multiple Sclerosis. Hum. Brain Mapp. 2005. [CrossRef] [PubMed]

66. Loitfelder, M.; Fazekas, F.; Koschutnig, K.; Fuchs, S.; Petrovic, K.; Ropele, S.; Pichler, A.; Jehna, M.; Langkammer, C.; Schmidt, R.; et al. Brain Activity Changes in Cognitive Networks in Relapsing-Remitting Multiple Sclerosis-Insights from a Longitudinal FMRI Study. PLoS ONE 2014. [CrossRef] [PubMed]

67. Staffen, W.; Mair, A.; Zauner, H.; Unterrainer, J.; Niederhofer, H.; Kutzelnigg, A.; Ritter, S.; Golaszewski, S.; Iglseder, B.; Ladurner, G. Cognitive Function and FMRI in Patients with Multiple Sclerosis: Evidence for Compensatory Cortical Activation during an Attention Task. Brain 2002. [CrossRef]

68. Clark, D.J. Automaticity of Walking: Functional Significance, Mechanisms, Measurement and Rehabilitation Strategies. Front. Hum. Neurosci. 2015. [CrossRef] [PubMed]

69. Kazui, H.; Kitagaki, H.; Mori, E. Cortical Activation during Retrieval of Arithmetical Facts and Actual Calculation: A Functional Magnetic Resonance Imaging Study. Psychiatry Clin. Neurosci. 2000, 54, 479-485. [CrossRef] [PubMed]

70. Dietrich, A. Transient Hypofrontality as a Mechanism for the Psychological Effects of Exercise. Psychiatry Res. 2006. [CrossRef] [PubMed]

71. Verghese, J.; Holtzer, R.; Lipton, R.B.; Wang, C. Mobility Stress Test Approach to Predicting Frailty, Disability, and Mortality in High-Functioning Older Adults. J. Am. Geriatr. Soc. 2012. [CrossRef]

72. Beurskens, R.; Bock, O. Age-Related Deficits of Dual-Task Walking: A Review. Neural Plast. 2012, 2012, 1-9. [CrossRef]

73. Holtzer, R.; Izzetoglu, M. Mild Cognitive Impairments Attenuate Prefrontal Cortex Activations during Walking in Older Adults. Brain Sci. 2020, 10, 415. [CrossRef] [PubMed]

74. Donoghue, O.A.; Cronin, H.; Savva, G.M.; O'Regan, C.; Kenny, R.A. Effects of Fear of Falling and Activity Restriction on Normal and Dual Task Walking in Community Dwelling Older Adults. Gait Posture 2013, 38, 120-124. [CrossRef] [PubMed]

75. Murphy, S.L.; Williams, C.S.; Gill, T.M. Characteristics Associated with Fear of Falling and Activity Restriction in CommunityLiving Older Persons. J. Am. Geriatr. Soc. 2002. [CrossRef]

76. Schoene, D.; Heller, C.; Aung, Y.N.; Sieber, C.C.; Kemmler, W.; Freiberger, E. A Systematic Review on the Influence of Fear of Falling on Quality of Life in Older People: Is There a Role for Falls? Clin. Interv. Aging. 2019. [CrossRef] [PubMed]

77. Vellas, B.J.; Wayne, S.J.; Romero, L.; Baumgartner, R.N.; Rubenstein, L.Z.; Garry, P.J. One-Leg Balance Is an Important Predictor of Injurious Falls in Older Persons. J. Am. Geriatr. Soc. 1997. [CrossRef] [PubMed]

78. Wijlhuizen, G.J.; de Jong, R.; Hopman-Rock, M. Older Persons Afraid of Falling Reduce Physical Activity to Prevent Outdoor Falls. Prev. Med. 2007. [CrossRef] [PubMed]

79. Herold, F.; Müller, P.; Gronwald, T.; Müller, N.G. Dose-Response Matters!-A Perspective on the Exercise Prescription in Exercise-Cognition Research. Front. Psychol. 2019. [CrossRef] [PubMed] 
80. Wollesen, B.; Wanstrath, M.; Van Schooten, K.S.; Delbaere, K. A Taxonomy of Cognitive Tasks to Evaluate Cognitive-Motor Interference on Spatiotemoporal Gait Parameters in Older People: A Systematic Review and Meta-Analysis. Eur. Rev. Aging Phys. Act. 2019. [CrossRef]

81. Izzetoglu, M.; Holtzer, R. Effects of Processing Methods on FNIRS Signals Assessed during Active Walking Tasks in Older Adults. IEEE Trans. Neural Syst. Rehabil. Eng. 2020, 28, 699-709. [CrossRef]

82. Menant, J.C.; Maidan, I.; Alcock, L.; Al-Yahya, E.; Cerasa, A.; Clark, D.J.; de Bruin, E.; Fraser, S.; Gramigna, V.; Hamacher, D.; et al. A Consensus Guide to Using Functional Near-Infrared Spectroscopy in Posture and Gait Research. Gait Posture 2020. [CrossRef] [PubMed]

83. Yucel, M.A.; Luhmann, A.V.; Scholkmann, F.; Gervain, J.; Dan, I.; Ayaz, H.; Boas, D.; Cooper, R.J.; Culver, J.; Elwell, C.E.; et al. Best Practices for fNIRS publications. Neurophotonics 2021. [CrossRef]

84. McKendrick, R.; Parasuraman, R.; Murtza, R.; Formwalt, A.; Baccus, W.; Paczynski, M.; Ayaz, H. Into the Wild: Neuroergonomic Differentiation of Hand-Held and Augmented Reality Wearable Displays during Outdoor Navigation with Functional Near Infrared Spectroscopy. Front. Hum. Neurosci. 2016. [CrossRef] [PubMed]

85. McKendrick, R.; Mehta, R.; Ayaz, H.; Scheldrup, M.; Parasuraman, R. Prefrontal Hemodynamics of Physical Activity and Environmental Complexity during Cognitive Work. Hum. Factors 2017. [CrossRef] [PubMed]

86. Dehais, F.; Karwowski, W.; Ayaz, H. Brain at Work and in Everyday Life as the Next Frontier: Grand Field Challenges for Neuroergonomics. Front. Neuroergonom. 2020. [CrossRef]

87. Ayaz, H.; Dehais, F. Neuroegonomics: The Brain at Work and in Everyday Life, 1st ed.; Academic Press: London, UK, 2019. [CrossRef] 